\title{
Prognostic Significance of Carbohydrate Antigen 125 in Acute Heart Failure: A Prospective Comparative Study With N-terminal Pro-B-type Natriuretic Peptide
}

\section{Ji Zhang ( $\sim$ js_zhangji@126.com)}

Wujin Hospital Affiliated with Jiangsu University, the Wujin Clinical College of Xuzhou Medical University Wenhua Li

Wujin Hospital Affiliated with Jiangsu University, the Wujin Clinical College of Xuzhou Medical University Gaojun Cai

Wujin Hospital Affiliated with Jiangsu University, the Wujin Clinical College of Xuzhou Medical University Jianqiang Xiao

Wujin Hospital Affiliated with Jiangsu University, the Wujin Clinical College of Xuzhou Medical University Jie Hui

The First Affiliated Hospital of Soochow University

\section{Ganwei Shi}

Wujin Hospital Affiliated with Jiangsu University, the Wujin Clinical College of Xuzhou Medical University Lei Yu

Wujin Hospital Affiliated with Jiangsu University, the Wujin Clinical College of Xuzhou Medical University Sheliang Xue

Wujin Hospital Affiliated with Jiangsu University, the Wujin Clinical College of Xuzhou Medical University

\section{Research Article}

Keywords: carbohydrate antigen 125 , risk prediction, $\mathrm{N}$-terminal pro-B-type natriuretic peptide, acute heart failure

Posted Date: September 29th, 2021

DOI: https://doi.org/10.21203/rs.3.rs-864855/v2

License: (9) (1) This work is licensed under a Creative Commons Attribution 4.0 International License. Read Full License 


\section{Abstract}

Background: In acute heart failure (AHF), elevated carbohydrate antigen 125 (CA125) and N-terminal proB-type natriuretic peptide (NTproBNP) have shown to correlate with adverse events. We sought to quantify their prognostic usefulness in predicting the 6-month combined endpoint of death/heart failure readmission.

Methods: The study includes 352 patients admitted for AHF. The primary endpoint was 6-month combined endpoint of death/AHF rehospitalization. CA125 and NTproBNP were dichotomized according to the best cut-offs to predict 6-month primary endpoint. By multivariate Cox regression analysis, the independent association of CA125 and NTproBNP with the primary endpoint was assessed, and their incremental prognostic utility evaluated by net reclassification improvement (NRI) and integrated discrimination improvement (IDI) index.

Results: A total of 47 (13.4\%) deaths and 113 (32.1\%) AHF rehospitalizations were identified at 6-month follow-up. The subjects with CA125 $\geq 39.7 \mathrm{U} / \mathrm{ml}$ and NTproBNP $\geq 3900 \mathrm{pg} / \mathrm{ml}$ had significantly higher cumulative event rates ( $56.1 \%$ vs. $33.3 \%$ and $53.3 \%$ vs. $33.8 \%$, both $\mathrm{P}<0.001)$. Elevated CA125 (HR 1.93; $95 \% \mathrm{Cl}$ [1.32-2.83]; $\mathrm{P}=0.001)$ was associated with higher $\mathrm{HR}$ than NTproBNP $\geq 3900 \mathrm{pg} / \mathrm{ml}(\mathrm{HR} 1.71 ; 95 \% \mathrm{Cl}$ [1.19-2.48]; $P=0.004)$ after adjusting for established risk factors. Elevated CA125 still independently predicted adverse events when both CA125 and NTproBNP were entered together in the same multivariate model. Furthermore, risk reclassification analyses demonstrated significant improvements in NRI of 22.3\% ( $P=0.014)$ and IDI of 2.7\% ( $P=0.012)$ when adding CA125 to the base model + NTproBNP.

Conclusions: Elevated CA125 and NTproBNP predicted adverse outcomes in AHF patients. CA125 added prognostic value to NTproBNP, and thus, their combination conferred greater predictive capacity.

\section{Introduction}

Given the multiple co-morbidities in acute heart failure (AHF) patients, risk prediction remains challenging. Identifying high-risk subjects help to further management by optimizing diuretic therapy, increasing the frequency of monitoring visits and so on.

Published studies indicate that high levels of several biomarkers ${ }^{1}$, including natriuretic peptides ${ }^{2,3}$, ST- ${ }^{4-}$

6 , cardiac troponins ${ }^{7,8}$, and carbohydrate antigen 125 (CA125) ${ }^{9,10}$ correlate with AHF severity and adverse outcomes. On the basis of different pathophysiological pathways involving heart failure progression and response patterns for the modification over time, we speculate that integrating multiple biomarkers will improve prognostic power in subjects admitted for AHF. As a widely used biomarker for monitoring ovarian cancer ${ }^{11}$, CA125 has been studied in heart diseases patients ${ }^{10,12-14}$ and especially in heart failure ${ }^{10,12,15}$, emerging as a surrogate for fluid overload and/or cytokine production in AHF ${ }^{16}$.

Our study aimed to evaluate CA125's prognostic utility in predicting 6-month combined endpoint of death/AHF rehospitalization among AHF patients. 


\section{Methods}

\section{Study population and design}

This was a prospective, observational cohort study from a single center that included 352 patients consecutively admitted to the cardiology ward from December 2019 to September 2020 due to AHF following current guidelines ${ }^{17,18}$. AHF was the main diagnosis of hospitalization for the purpose of our study. Patients with a diagnosis of severe hepatic disease, sepsis, ongoing dialysis treatment for endstage renal disease, pulmonary embolism, or acute rheumatic and autoimmune diseases were excluded by design. Demographic information, vital signs, medications, medical history were collected, along with standard echocardiographic evaluation, laboratory results and 12-lead electrocardiogram during index admission. Intravenous furosemide or torasemide was used in all patients at least $24 \mathrm{~h}$ after admission. Treatment guidelines established were followed ${ }^{17-19}$. Time to death/AHF readmission whichever occurred first was the primary endpoint at 6 months follow-up.

Subjects were followed up through outpatient service or by telephone. Patients were censored free of event or lost to follow-up at last contact within this period or at 6-month. The local ethics committee approved this study, and all patients provided informed consent to their participation in accordance with the Declaration of Helsinki.

\section{Biomarkers measurement}

CA125 serum level was obtained between $5: 30$ and 8:00 $\mathrm{h}$ on the 2 nd day of admission, while N-terminal pro-B-type natriuretic peptide (NTproBNP) serum level was immediately determined after admission using commercially available immunoassay kits (Elecsys CA125 II assay, Roche Diagnostics and Vitros Immunodiagnostic Products NT-proBNP Reagent Pack, Ortho-Clinical Diagnostics, respectively). A blinded technician to clinical information performed the biomarker's assay.

\section{Statistical analysis}

Categorical variables were presented as frequencies and percentages, continuous variables summarized as mean \pm standard deviation or median (interquartile range). We dichotomized both biomarkers according to the best predictive cut-offs and compared between-group baseline characteristics using t test, Mann-Whitney test, chi-square or Fisher exact test, as appropriate. The resulting cut-off values were $39.7 \mathrm{U} / \mathrm{ml}$ for CA125 and $3900 \mathrm{pg} / \mathrm{ml}$ for NTproBNP. The cumulative rate of events (death or AHF readmission) among CA125 or NTproBNP categories were estimated and compared using the Kaplan-Meier method and log-rank test. The relation of CA125 and NTproBNP with the primary endpoint were determined by univariate and multivariate Cox analyses. Candidate variables in the initial multivariate model included clinical characteristics such as age, gender, weight, history of atrial fibrillation, diabetes, hypertension and acute myocardial infarction on admission. Biochemical variables included were serum creatinine, blood haemoglobin, serum sodium. We also included left ventricular ejection fraction (LVEF>50\% [reference], 36\%-50\%, and $\leq 35 \%$ ), admission heart 
rate, admission systolic blood pressure, evidence of pleural effusion, peripheral oedema, AHF category (acute decompensate heart failure [ADHF] or new-onset heart failure) in our analyses. For multivariate Cox regression analyses, we retained factors with $\mathrm{P}<0.15$ in univariate Cox analysis and those clinically relevant. Given number of events available, included variables were carefully chosen and a parsimonious multivariate Cox model was derived. CA125, NTproBNP, or both biomarkers were first entered individually in the multivariate model. The Schoenfeld residuals was used to test the proportional hazards assumption over time.

Harrell's C-statistics measured discriminative ability of the models. CA125's incremental prognostic utility to NTproBNP and baseline variables was evaluated using integrated discrimination improvement (IDI) and net reclassification improvement (NRI) with the corresponding P values. We performed two multiple linear analyses to examine relations of log-transformed CA125 and NTproBNP to clinical variables.

In all analyses, 2-sided P-value of $<0.05$ was considered statistically significant. The main analysis was performed using SPSS 26.0. Risk reclassifification was calculated in R 4.0.3.

\section{Results}

\section{Baseline characteristics}

Of 352 subjects, $49.4 \%$ had LVEF $>50 \%$. The sample was $46.9 \%$ female and the mean age was $76 \pm 11$ years. Median baseline levels of the entire population for CA125 and NTproBNP were $43.2 \mathrm{U} / \mathrm{ml}$ (21.6$102.7)$ and $5170 \mathrm{pg} / \mathrm{ml}$ (2748-10000), respectively. Baseline characteristics of study participants by CA125 and NTproBNP categories are shown in Tables 1 and 2. Patients with both biomarkers elevated $(\mathrm{CA} 125 \geq 39.7 \mathrm{U} / \mathrm{ml}$ and NTproBNP $\geq 3900 \mathrm{pg} / \mathrm{ml}$ ) exhibited a worse clinical profile. Lower LVEF and pleural effusion were more prevalent when both biomarkers were elevated. 
Table 1

Baseline characteristics stratified by CA125 categories

\begin{tabular}{|c|c|c|c|}
\hline & $\begin{array}{l}\text { CA125『39.7U/ml }(n= \\
159)\end{array}$ & $\begin{array}{l}\mathrm{CA} 125 \geq 39.7 \mathrm{U} / \mathrm{ml}(\mathrm{n}= \\
193)\end{array}$ & $\begin{array}{l}P- \\
\text { value }\end{array}$ \\
\hline \multicolumn{4}{|l|}{ Demographic and medical history } \\
\hline Age, years & $77 \pm 9$ & $75 \pm 12$ & 0.085 \\
\hline Female, n (\%) & $83(52.2)$ & $82(42.5)$ & 0.069 \\
\hline Weight, kg & $60.4 \pm 12.3$ & $59.4 \pm 12.1$ & 0.425 \\
\hline Hypertension, n (\%) & 108 (67.9) & $112(58.0)$ & 0.056 \\
\hline Diabetes mellitus, n (\%) & $44(27.7)$ & $55(28.5)$ & 0.864 \\
\hline Atrial fibrillation, n (\%) & $76(47.8)$ & $107(55.4)$ & 0.153 \\
\hline $\begin{array}{l}\text { Previous coronary artery disease, } \mathrm{n} \\
(\%)\end{array}$ & $38(23.9)$ & $33(17.1)$ & 0.114 \\
\hline $\begin{array}{l}\text { Previous myocardial infarction, } \mathrm{n} \\
(\%)\end{array}$ & $19(11.9)$ & $15(7.8)$ & 0.187 \\
\hline Acute myocardial infarction, n (\%) & $25(15.7)$ & $11(5.7)$ & 0.002 \\
\hline Previous PCl, n (\%) & $15(9.4)$ & $9(4.7)$ & 0.077 \\
\hline Valvular heart disease, n (\%) & $17(10.7)$ & $24(12.4)$ & 0.612 \\
\hline ADHF, n (\%) & $131(82.4)$ & $182(94.3)$ & $\varangle 0.001$ \\
\hline Previous pacemaker, n (\%) & $4(2.5)$ & $5(2.6)$ & 1.000 \\
\hline Anemia, n (\%) & $43(27.0)$ & $51(26.4)$ & 0.896 \\
\hline Previous stroke, $\mathrm{n}(\%)$ & $17(10.7)$ & $22(11.4)$ & 0.833 \\
\hline COPD, n (\%) & $35(22.0)$ & $45(23.3)$ & 0.771 \\
\hline Previous malignancy, n (\%) & $2(1.3)$ & $12(6.2)$ & 0.018 \\
\hline Pleural effusion, n (\%) & $59(37.1)$ & $140(72.5)$ & $\otimes 0.001$ \\
\hline Peripheral oedema, n (\%) & $38(23.9)$ & $96(49.7)$ & $₫ 0.001$ \\
\hline \multicolumn{4}{|l|}{ Vital signs } \\
\hline Heart rate, b.p.m. & $87 \pm 22$ & $93 \pm 24$ & 0.033 \\
\hline Systolic blood pressure, $\mathrm{mmHg}$ & $136 \pm 24$ & $135 \pm 23$ & 0.564 \\
\hline Diastolic blood pressure, mmHg & $80 \pm 16$ & $83 \pm 15$ & 0.150 \\
\hline
\end{tabular}




\begin{tabular}{|c|c|c|c|}
\hline & $\begin{array}{l}\text { CA125区39.7U/ml }(n= \\
159)\end{array}$ & $\begin{array}{l}\mathrm{CA} 125 \geq 39.7 \mathrm{U} / \mathrm{ml}(\mathrm{n}= \\
193)\end{array}$ & $\begin{array}{l}\mathrm{P}- \\
\text { value }\end{array}$ \\
\hline Haemoglobin (g/L) & $123.6 \pm 24.0$ & $126.3 \pm 25.6$ & 0.314 \\
\hline Serum creatinine (umol/L) & $86(69-117)$ & $83(66-114)$ & 0.340 \\
\hline Sodium (mmol/L) & $140.1 \pm 5.3$ & $139.0 \pm 5.3$ & 0.057 \\
\hline NTproBNP (pg/ml) & $4200(2510-7940)$ & $5990(3245-11400)$ & 0.002 \\
\hline CA125 (U/ml) & $21(13-27)$ & $91(56-173)$ & $\nabla 0.001$ \\
\hline \multicolumn{4}{|l|}{ Echocardiography } \\
\hline LVEF (\%) & $50 \pm 13$ & $47 \pm 13$ & 0.020 \\
\hline LVEF $\leq 35 \%, \mathrm{n}(\%)$ & $27(17.0)$ & $41(21.2)$ & 0.313 \\
\hline LVEF $\leq 50 \%, n(\%)$ & $43(27.0)$ & $67(34.7)$ & 0.122 \\
\hline LVDD (mm) & $53 \pm 9$ & $53 \pm 10$ & 0.802 \\
\hline LVSD (mm) & $39 \pm 10$ & $40 \pm 11$ & 0.317 \\
\hline LAD (mm) & $46 \pm 8$ & $48 \pm 9$ & 0.035 \\
\hline \multicolumn{4}{|c|}{$\begin{array}{l}\text { PCI, percutaneous coronary intervention; COPD, chronic obstructive pulmonary disease; LVDD, left } \\
\text { ventricular diastolic diameter; LVSD, left ventricular systolic diameter; LAD, left atrial diameter. }\end{array}$} \\
\hline \multicolumn{4}{|c|}{ Anemia, defined as a hemoglobin level $<120 \mathrm{~g} / \mathrm{L}$ in men and $<110 \mathrm{~g} / \mathrm{L}$ in women. } \\
\hline \multicolumn{4}{|c|}{ Values are mean $\pm S D, n(\%)$, or median (interquartile range). } \\
\hline
\end{tabular}


Table 2

Baseline characteristics stratified by NTproBNP categories

NTproBNP®3900pg/ml (n NTproBNP $\geq 3900 \mathrm{pg} / \mathrm{ml}$ (n

$=138$ )

$=214$ )

Pvalue

Demographic and medical

history

\begin{tabular}{|c|c|c|c|}
\hline Age, years & $76 \pm 10$ & $75 \pm 12$ & 0.741 \\
\hline Female, $\mathrm{n}(\%)$ & $71(51.4)$ & $94(43.9)$ & 0.167 \\
\hline Weight, kg & $62.3 \pm 12.8$ & $58.2 \pm 11.5$ & 0.002 \\
\hline Hypertension, n(\%) & $90(65.2)$ & $130(60.7)$ & 0.398 \\
\hline Diabetes mellitus, n(\%) & $45(32.6)$ & $54(25.2)$ & 0.133 \\
\hline Atrial fibrillation, $\mathrm{n}(\%)$ & $82(59.4)$ & $101(47.2)$ & 0.025 \\
\hline $\begin{array}{l}\text { Previous coronary artery } \\
\text { disease, } \mathrm{n}(\%)\end{array}$ & $24(17.4)$ & $47(22.0)$ & 0.297 \\
\hline $\begin{array}{l}\text { Previous myocardial infarction, } \\
\mathrm{n}(\%)\end{array}$ & $12(8.7)$ & $22(10.3)$ & 0.623 \\
\hline $\begin{array}{l}\text { Acute myocardial infarction, } \\
\mathrm{n}(\%)\end{array}$ & $11(8.0)$ & $25(11.7)$ & 0.262 \\
\hline Previous PCI, n(\%) & $9(6.5)$ & $15(7.0)$ & 0.859 \\
\hline Valvular heart disease, $\mathrm{n}(\%)$ & $13(9.4)$ & $28(13.1)$ & 0.296 \\
\hline ADHF, n (\%) & $126(91.3)$ & $187(87.4)$ & 0.253 \\
\hline Previous pacemaker, n(\%) & $3(2.2)$ & $6(2.8)$ & 0.984 \\
\hline Anemia, n(\%) & $32(23.2)$ & $62(29.0)$ & 0.231 \\
\hline Previous stroke, $n(\%)$ & $10(7.2)$ & $29(13.6)$ & 0.066 \\
\hline COPD, n(\%) & $38(27.5)$ & $42(19.6)$ & 0.084 \\
\hline Previous malignancy, $\mathrm{n}(\%)$ & $3(2.2)$ & $11(5.1)$ & 0.164 \\
\hline Pleural effusion, n (\%) & $62(44.9)$ & $137(64.0)$ & $\begin{array}{l}< \\
0.001\end{array}$ \\
\hline Peripheral oedema, n (\%) & $52(37.7)$ & $82(38.3)$ & 0.904 \\
\hline \multicolumn{4}{|l|}{ Vital signs } \\
\hline Heart rate, b.p.m. & $88 \pm 23$ & $91 \pm 24$ & 0.220 \\
\hline Systolic blood pressure, $\mathrm{mmHg}$ & $136 \pm 22$ & $135 \pm 24$ & 0.753 \\
\hline
\end{tabular}




\begin{tabular}{|c|c|c|c|}
\hline & $\begin{array}{l}\text { NTproBNP®3900pg/ml (n } \\
=138)\end{array}$ & $\begin{array}{l}\text { NTproBNP } \geq 3900 \mathrm{pg} / \mathrm{ml}(\mathrm{n} \\
=214)\end{array}$ & $\begin{array}{l}\mathrm{P}- \\
\text { value }\end{array}$ \\
\hline $\begin{array}{l}\text { Diastolic blood pressure, } \\
\mathrm{mmHg}\end{array}$ & $80 \pm 14$ & $83 \pm 17$ & 0.106 \\
\hline \multicolumn{4}{|l|}{ Laboratory } \\
\hline Haemoglobin (g/L) & $126.6 \pm 24.1$ & $124.1 \pm 25.3$ & 0.355 \\
\hline Serum creatinine (umol/L) & $76(60-94)$ & $90(71-131)$ & $\begin{array}{l}< \\
0.001\end{array}$ \\
\hline Sodium (mmol/L) & $139.3 \pm 6.3$ & $139.7 \pm 4.6$ & 0.507 \\
\hline NTproBNP (pg/ml) & $2435(1728-3093)$ & $8590(5728-13025)$ & $\begin{array}{l}< \\
0.001\end{array}$ \\
\hline CA125 (U/ml) & $34(19-65)$ & $54(24-117)$ & 0.001 \\
\hline \multicolumn{4}{|l|}{ Echocardiography } \\
\hline LVEF (\%) & $53 \pm 11$ & $45 \pm 13$ & $\begin{array}{l}< \\
0.001\end{array}$ \\
\hline LVEF $\leq 35 \%, n(\%)$ & $14(10.1)$ & $54(25.2)$ & $\begin{array}{l}< \\
0.001\end{array}$ \\
\hline LVEF $\leq 50 \%, n(\%)$ & $36(26.1)$ & $74(34.6)$ & 0.093 \\
\hline LVDD (mm) & $50 \pm 10$ & $54 \pm 10$ & $<.001$ \\
\hline LVSD mm & $36 \pm 10$ & $42 \pm 11$ & $\begin{array}{l}< \\
0.001\end{array}$ \\
\hline LAD (mm) & $46 \pm 9$ & $47 \pm 8$ & 0.412 \\
\hline \multicolumn{4}{|l|}{ Abbreviations as in Table 1.} \\
\hline \multicolumn{4}{|c|}{ Anemia, defined as a hemoglobin level $<120 \mathrm{~g} / \mathrm{L}$ in men and $<110 \mathrm{~g} / \mathrm{L}$ in women. } \\
\hline \multicolumn{4}{|c|}{ Values are mean $\pm S D, n(\%)$, or median (interquartile range). } \\
\hline
\end{tabular}

\section{Clinical predictors of CA125 and NTproBNP}

Table 3 listed those variables independently correlated with log-transformed CA125 and NTproBNP. We identified different clinical predictors of these two biomarkers in the setting of AHF. For InCA125 the most important predictors were pleural effusion and ADHF (standardized $\beta$ coefficients 0.392 and 0.231 , respectively). The most important predictors of InNTproBNP were serum creatinine, weight and LVEF (standardized $\beta$ coefficients $0.382,-0.306$ and -0.286 , respectively). 
Ln (CA125)

Pleural effusion

$\bigotimes 0.001$

ADHF

0.231

$\bigotimes 0.001$

Peripheral oedema

0.173

$\bigotimes 0.001$

Weight, kg

$-0.154$

0.002

Age, years

$-0.151$

0.003

LVEF

$-0.132$

0.006

Sodium

$-0.106$

0.018

Ln (NTproBNP)

Serum creatinine

0.382

$₫ 0.001$

Weight, kg

$-0.306$

$₫ 0.001$

LVEF

$-0.286$

$\varangle 0.001$

Pleural effusion

0.154

$凶 0.001$

ADHF

$-0.141$

0.001

LVDD

0.139

0.019

Ln(CA125), antigen carbohydrate 125 natural logarithm; Ln(NTproBNP), N-terminal pro-B-type natriuretic peptide natural logarithm; LVDD, left ventricular diastolic diameter.

Moreover, we found differential associations of CA125 and NTproBNP with clinical presentations of AHF. A presentation as ADHF was associated with higher CA125 levels; conversely admission for ADHF was independently and positively related to NTproBNP values.

\section{CA125 levels, NTproBNP levels, and the primary endpoint}

In total, 47 patients (13.4\%) died (12 deaths occurred during the index admission and 35 post discharge), $113(32.1 \%)$ AHF rehospitalizations identified at 6-month follow-up. CA125 and NTproBNP values in subjects experiencing death/AHF rehospitalization were significantly higher when compared with those free of event (56.3 U/ml [27.2-135.6] vs. $33.9 \mathrm{U} / \mathrm{ml}$ [18.4-79.8] and $6255 \mathrm{pg} / \mathrm{ml}$ [3425-6255] vs. $4085 \mathrm{pg} / \mathrm{ml}$ [2390-8015], respectively, $\mathrm{P}<0.001$ for both).

By the Kaplan-Meier method, subjects with CA125 $\geq 39.7 \mathrm{U} / \mathrm{ml}$ and NTproBNP $\geq 3900 \mathrm{pg} / \mathrm{ml}$ exhibited significantly higher cumulative event rates $(56.1 \%$ vs. $33.3 \%$ and $53.3 \%$ vs. $33.8 \%$, both $P<0.001$, Fig. $1 \mathrm{~A}$, 
B). When combined (Fig. 1C), patients with both biomarkers elevated had the highest cumulative event rate (61.5\%); intermediate when only one of them was elevated: $44.2 \%$ for those with only CA125 elevated and $40.5 \%$ for subjects with only NTproBNP elevated, respectively, and lower (25.3\%) for patients with values below the chosen biomarker cutpoints, $\mathrm{P}$ trend $<0.001$.

Table 4 displayed the results of univariate and multivariate modeling. In the multivariate Cox analysis, elevated CA125 (HR 1.93; 95\% CI [1.32-2.83]; $P=0.001$ ) was associated with higher adjusted HR than NTproBNP $\geq 3900 \mathrm{pm} / \mathrm{ml}(\mathrm{HR} 1.71 ; 95 \% \mathrm{Cl}$ [1.19-2.48]; $\mathrm{P}=0.004)$. Elevated CA125 still independently predicted adverse events when both CA125 and NTproBNP were entered together in the same multivariate model. In the final Cox model, serum creatinine and NTproBNP $\geq 3900 \mathrm{pm} / \mathrm{ml}$ were other independent predictors. When these two biomarkers included in the final Cox model, no interactions were found $(\mathrm{P}=$ 0.508). 
Table 4

CA125 and NTproBNP hazard ratios for 6-month combined endpoint of death/AHF readmission.

\begin{tabular}{|c|c|c|c|c|}
\hline Variables & Univariate & & Multivariate & \\
\hline & $\mathrm{HR}(95 \% \mathrm{Cl})$ & $\begin{array}{l}\mathrm{P} \text { - } \\
\text { value }\end{array}$ & $\mathrm{HR}(95 \% \mathrm{Cl})$ & $\begin{array}{l}\mathrm{P}- \\
\text { value }\end{array}$ \\
\hline Age (/10 years increase) & $\begin{array}{l}1.10(0.96- \\
1.29)\end{array}$ & 0.167 & $\begin{array}{l}1.06(0.90- \\
1.24)\end{array}$ & 0.457 \\
\hline Atrial fibrillation & $\begin{array}{l}1.38(1.01- \\
1.89)\end{array}$ & 0.046 & $\begin{array}{l}1.21(0.85- \\
1.71)\end{array}$ & 0.291 \\
\hline Serum creatinine (/SD increase) & $\begin{array}{l}1.20 \\
(1.00-1.01)\end{array}$ & 0.004 & $\begin{array}{l}1.20(1.04- \\
1.38)\end{array}$ & 0.014 \\
\hline LVEF $\leq 35 \%$ & $\begin{array}{l}0.76(0.48- \\
1.21)\end{array}$ & 0.251 & $\begin{array}{l}0.75(0.45- \\
1.27)\end{array}$ & 0.285 \\
\hline LVEF $\leq 50 \%$ & $\begin{array}{l}1.37(0.98- \\
1.92)\end{array}$ & 0.069 & $\begin{array}{l}1.30(0.92- \\
1.86)\end{array}$ & 0.142 \\
\hline $\begin{array}{l}\text { Systolic blood pressure (/10 mmHg } \\
\text { increase) }\end{array}$ & $0.93(0.87-1.00)$ & 0.042 & $\begin{array}{l}0.96(0.90- \\
1.03)\end{array}$ & 0.222 \\
\hline Sodium (/SD increase) & $\begin{array}{l}0.90(0.79- \\
1.03)\end{array}$ & 0.112 & $\begin{array}{l}0.92(0.79- \\
1.06)\end{array}$ & 0.243 \\
\hline ADHF & $\begin{array}{l}1.39(0.80- \\
2.40)\end{array}$ & 0.244 & $\begin{array}{l}1.15(0.63- \\
2.08)\end{array}$ & 0.647 \\
\hline Pleural effusion & $\begin{array}{l}1.32(0.96- \\
1.82)\end{array}$ & 0.086 & $\begin{array}{l}0.99(0.69- \\
1.42)\end{array}$ & 0.955 \\
\hline Peripheral oedema & $\begin{array}{l}1.40(1.04- \\
1.91)\end{array}$ & 0.034 & $\begin{array}{l}1.14(0.82- \\
1.60)\end{array}$ & 0.430 \\
\hline $\mathrm{CA} 125 \geq 39.7 \mathrm{U} / \mathrm{ml}$ & $\begin{array}{l}2.00(1.44- \\
2.79)\end{array}$ & $<.001$ & $\begin{array}{l}1.78(1.22- \\
2.61)\end{array}$ & 0.003 \\
\hline NTproBNP $\geq 3900 \mathrm{pg} / \mathrm{ml}$ & $\begin{array}{l}1.78(1.26- \\
2.50)\end{array}$ & 0.001 & $\begin{array}{l}1.57(1.08- \\
2.27)\end{array}$ & 0.018 \\
\hline
\end{tabular}

We compared the performance of each regression model by using Harrell's C-statistic as a discrimination measure. Compared with the model including NTproBNP alone (0.623), CA125 alone (0.635) or none (0.606), the Cox model including CA125 and NTproBNP had a higher C-statistic (0.648). IDI and NRI values were significantly higher when adding each biomarker, or both to the model containing baseline variables. Furthermore, a significant improvement in NRI of $22.3 \%(P=0.014)$ and to IDI of $2.7 \%(P=$ 0.012) was observed when adding CA125 to the base model + NTproBNP, supporting the incremental prognostic effect on top of NTproBNP (Table 5). 
Table 5

Reclassification results for 6-month combined endpoint of death/AHF rehospitalization.

\begin{tabular}{|lll|}
\hline & NRI (\%) (P-value) & IDI (\%) (P-value) \\
\hline Model 2 vs. 1 & $16.2(0.014)$ & $2.6(0.010)$ \\
\hline Model 3 vs. 1 & $23.8(0.008)$ & $3.5(0.002)$ \\
\hline Model 4 vs. 1 & $27.0(0.002)$ & $5.3(<0.001)$ \\
\hline Model 4 vs. 2 & $22.3(0.024)$ & $2.7(0.020)$ \\
\hline NRI, net reclassifification & improvement; IDI, integrated discrimination improvement. \\
Model 1 = base model. & \\
Model 2 = base model + NTproBNP categories. & \\
Model 3 = base model + CA125 categories. & \\
Model 4 = base model + NTproBNP categories + CA125 categories.
\end{tabular}

\section{Discussion}

Our study compared the risk prediction capacity of NTproBNP and CA125 in the setting of AHF. After multivariate adjustment, elevation of CA125 and NTproBNP had negative prognostic effect on event-free survival. Not only elevated NTproBNP but also CA125 remained independent predictors of poor outcomes by combined both biomarkers. Additionally, adding CA125 in the model including NTproBNP significantly improved predictive power.

Congestion as a strong predictor of heart failure-related readmission and death ${ }^{20}$, being responsible for most of heart failure decompensation, is an important therapeutic target in AHF ${ }^{17,18}$; however, evaluation of congestion remains a challenge in the routine management of $\mathrm{AHF}^{21}$. Perhaps the limited accuracy of signs and symptoms for quantifying fluid overload severity ${ }^{22,23}$, signs of congestion (peripheral oedema, pleural effusion and so on) are not routinely used for risk stratification. Suitable biomarkers would optimize risk prediction. CA125 levels correlate well with signs of fluid congestion $9,10,16$ and pulmonary artery wedge pressure ${ }^{10,16}$. In this study, the most important clinical predictor of serum CA125 levels was the presence of pleural effusion. As a marker of congestion, CA125 being related with adverse events in heart failure patients ${ }^{9,10}$, has been shown to be indicative as a heart failure severity surrogate. Elevated CA125 is an independent predictor with incremental prognostic value over traditional prognosticators and natriuretic peptides ${ }^{9}$, and thus, combining both biomarkers improved risk stratification in $\mathrm{AHF}^{10}$.

Interestingly, although CA125 has shown to be a potential tool for treatment guiding in $\mathrm{AHF}^{12,24}$, little support is available regarding the benefits of NP-guided therapy over usual care ${ }^{25}$. In the CHANCE-HF trial, compared to the standard of care, a CA125-guided therapy characterized by a higher frequency of 
furosemide equivalent dose adjustments and ambulatory intravenous furosemide administrations according to CA125 response and clinical profile indicated a significantly reduced risk of 1-year mortality or AHF readmission ${ }^{12}$. In a recent multicenter randomized study of 160 AHF subjects with renal dysfunction, a CA125-guided diuretic strategy with admission loop diuretics dose determined on the basis of CA125 levels significantly improved 72-h eGFR ${ }^{24}$. Briefly, in subjects with high CA125 levels, highintensity diuretic treatment and/or closer follow-up were advocated. When CA125 was low or decreased, a down-titration was recommended in both trials which endorsed the role of CA125-guided decongestion treatment in AHF.

Given CA125's long half-life (around 5-12 days) ${ }^{16}$, and a shorter mean half-life of NTproBNP (60-120 $\mathrm{min})^{26}, \mathrm{CA} 125$ potentially provides pathophysiological information several weeks prior and NTproBNP could provide acute haemodynamic information, being similar to glycated hemoglobin and serum glucose in diabetes. One study reported that levels of CA125 and NTproBNP represent distinct pathophysiological states related to heart failure severity ${ }^{10}$. The combined use of CA125 and NTproBNP improved risk stratification and this multi-marker approach hold promise in guiding depletive therapy, showing the need to incorporate CA125 into clinical daily practice. In addition, conversely to natriuretic peptides, age, gender, body weight and renal function did not significantly influence CA125 levels ${ }^{12,21}$. In current study, we found that NTproBNP strongly depended on serum creatinine, weight, and LVEF, while CA125 appeared not to be significantly influenced by other factors which are highly prevalent. Beyond these considerations, additional benefits for implementing CA125 testing in daily clinical practice arise from its standardized measurement, low cost, and wide availability.

Our study had some limitations. Firstly, its observational design makes it susceptible to confounding factors and bias. Secondly, it is a single-center study which precludes extrapolation of results. Thirdly, it is not possible to extrapolate findings to patients undergoing renal dialysis because this study included patients with baseline serum creatinine values $\leq 360 \mathrm{umol} / \mathrm{L}$. Finally, we measured CA125 levels at onetime point after an overnight fast on the second day of admission; however, peak CA125 levels might better reflect fluid overload in patients with AHF.

\section{Conclusions}

In AHF patients, elevated CA125 levels were highly predictive of 6-month death/AHF readmission, adding prognostic value to NTproBNP and clinical risk factors. Measuring these two biomarkers simultaneously conferred greater predictive capacity, when compared with either of them alone. Hence, this glycoprotein should be considered as a complement for optimal risk prediction. The underlying mechanisms of CA125 in AHF syndromes remain unclear and more research is needed.

\section{Abbreviations}

CA125, antigen carbohydrate 125; NTproBNP, N-terminal pro-B-type natriuretic peptide; LVEF, left ventricular ejection fraction; $A H F$, acute heart failure; $A D H F$, acute decompensate heart failure. 


\section{Declarations}

\section{Ethics approval and consent to participate}

the ethics committee of Wujin Hospital Affiliated with Jiangsu University approved this study, and all patients provided informed consent to their participation.

\section{Consent for publication}

Not applicable

\section{Availability of data and materials}

The datasets were analysed in this study available from the corresponding author on reasonable request.

\section{Competing interests}

The authors have no conflicts of interest to declare.

\section{Funding}

This study was supported by the Jiangsu Youth Medical Talents Project (QNRC2016310), Changzhou Science and Technology Support Project (social development) (CE20195003). No funding body participated in the design of the study and collection, analysis, and interpretation of data and in writing the manuscript.

\section{Authors' contributions}

JZ, WHL, JQX, JH wrote and designed the study. GJC was the statistical consultant. JZ and WHL wrote and revised the manuscript. GWS, LY and SLX collected data. All authors read and approved the final manuscript.

\section{Acknowledgements}

Not applicable.

\section{References}

1. Levy WC, Mozaffarian D, Linker DT, et al. The Seattle Heart Failure Model: prediction of survival in heart failure. Circulation. 2006;113(11):1424-1433. doi:10.1161/CIRCULATIONAHA.105.584102.

2. Stienen S, Salah K, Eurlings LW, et al. Challenging the two concepts in determining the appropriate pre-discharge N-terminal pro-brain natriuretic peptide treatment target in acute decompensated heart failure patients: absolute or relative discharge levels? Eur J Heart Fail. 2015;17(9):936-944.

doi:10.1002/ejhf.320. 
3. Kociol RD, Horton JR, Fonarow GC, et al. Admission, discharge, or change in B-type natriuretic peptide and long-term outcomes: data from Organized Program to Initiate Lifesaving Treatment in Hospitalized Patients with Heart Failure (OPTIMIZE-HF) linked to Medicare claims. Circ Heart Fail. 2011;4(5):628-636. doi:10.1161/CIRCHEARTFAILURE.111.962290.

4. Aleksova A, Paldino A, Beltrami AP, et al. Cardiac Biomarkers in the Emergency Department: The Role of Soluble ST2 (SST2) in Acute Heart Failure and Acute Coronary Syndrome-There is Meat on the Bone. J Clin Med. 2019;8(2):270. Published 2019 Feb 22. doi:10.3390/jcm8020270.

5. van Vark LC, Lesman-Leegte I, Baart SJ, et al. Prognostic Value of Serial ST2 Measurements in Patients With Acute Heart Failure. J Am Coll Cardiol. 2017;70(19):2378-2388. doi:10.1016/j.jacc.2017.09.026.

6. Morrow DA, Velazquez EJ, DeVore AD, et al. Cardiovascular biomarkers in patients with acute decompensated heart failure randomized to sacubitril-valsartan or enalapril in the PIONEER-HF trial. Eur Heart J. 2019;40(40):3345-3352. doi:10.1093/eurheartj/ehz240.

7. Peacock WF 4th, De Marco T, Fonarow GC, et al. Cardiac troponin and outcome in acute heart failure. N Engl J Med. 2008;358(20):2117-2126. doi:10.1056/NEJMoa0706824.

8. Felker GM, Mentz RJ, Teerlink JR, et al. Serial high sensitivity cardiac troponin T measurement in acute heart failure: insights from the RELAX-AHF study. Eur J Heart Fail. 2015;17(12):1262-1270. doi:10.1002/ejhf.341.

9. Núñez J, Bayés-Genís A, Revuelta-López E, et al. Clinical Role of CA125 in Worsening Heart Failure: A BIOSTAT-CHF Study Subanalysis. JACC Heart Fail. 2020;8(5):386-397. doi:10.1016/j.jchf.2019.12.005.

10. Núñez J, Sanchis J, Bodí V, et al. Improvement in risk stratification with the combination of the tumour marker antigen carbohydrate 125 and brain natriuretic peptide in patients with acute heart failure. Eur Heart J. 2010;31(14):1752-1763. doi:10.1093/eurheartj/ehq142.

11. Marcus CS, Maxwell GL, Darcy KM, Hamilton CA, McGuire WP. Current approaches and challenges in managing and monitoring treatment response in ovarian cancer. J Cancer. 2014;5(1):25-30. Published 2014 Jan 1. doi:10.7150/jca.7810.

12. Núñez J, Llàcer P, Bertomeu-González V, et al. Carbohydrate Antigen-125-Guided Therapy in Acute Heart Failure: CHANCE-HF: A Randomized Study. JACC Heart Fail. 2016;4(11):833-843. doi:10.1016/j.jchf.2016.06.007.

13. Rheude T, Pellegrini C, Schmid H, et al. Comparison of Carbohydrate Antigen 125 and N-Terminal ProBrain Natriuretic Peptide for Risk Prediction After Transcatheter Aortic Valve Implantation. Am J Cardiol. 2018;121(4):461-468. doi:10.1016/j.amjcard.2017.11.020.

14. Falcão F, Oliveira F, Cantarelli F, et al. Carbohydrate antigen 125 for mortality risk prediction following acute myocardial infarction. Sci Rep. 2020;10(1):11016. Published 2020 Jul 3. doi:10.1038/s41598020-67548-8.

15. Falcão F, de Oliveira FRA, da Silva MCFC, Sobral Filho DC. Carbohydrate antigen 125: a promising tool for risk stratification in heart diseases. Biomark Med. 2018;12(4):367-381. doi:10.2217/bmm- 
2017-0452.

16. Núñez J, Miñana G, Núñez E, Chorro FJ, Bodí V, Sanchis J. Clinical utility of antigen carbohydrate 125 in heart failure. Heart Fail Rev. 2014;19(5):575-584. doi:10.1007/s10741-013-9402-y.

17. Ponikowski P, Voors AA, Anker SD, et al. 2016 ESC Guidelines for the diagnosis and treatment of acute and chronic heart failure: The Task Force for the diagnosis and treatment of acute and chronic heart failure of the European Society of Cardiology (ESC)Developed with the special contribution of the Heart Failure Association (HFA) of the ESC. Eur Heart J. 2016;37(27):2129-2200. doi:10.1093/eurheartj/ehw128.

18. Yancy CW, Jessup M, Bozkurt B, et al. 2017 ACC/AHA/HFSA Focused Update of the 2013 ACCF/AHA Guideline for the Management of Heart Failure: A Report of the American College of Cardiology/American Heart Association Task Force on Clinical Practice Guidelines and the Heart Failure Society of America. Circulation. 2017;136(6):e137-e161.

doi:10.1161/CIR.0000000000000509.

19. Ibanez B, James S, Agewall S, et al. 2017 ESC Guidelines for the management of acute myocardial infarction in patients presenting with ST-segment elevation: The Task Force for the management of acute myocardial infarction in patients presenting with ST-segment elevation of the European Society of Cardiology (ESC). Eur Heart J. 2018;39(2):119-177. doi:10.1093/eurheartj/ehx393.

20. Rubio-Gracia J, Demissei BG, Ter Maaten JM, et al. Prevalence, predictors and clinical outcome of residual congestion in acute decompensated heart failure. Int J Cardiol. 2018;258:185-191. doi:10.1016/j.ijcard.2018.01.067.

21. Mullens W, Damman K, Harjola VP, et al. The use of diuretics in heart failure with congestion - a position statement from the Heart Failure Association of the European Society of Cardiology. Eur $J$ Heart Fail. 2019;21(2):137-155. doi:10.1002/ejhf.1369.

22. Parrinello G, Torres D, Paterna S, di Pasquale P, Licata G. The pathophysiology of acute heart failure: the key role of fluid accumulation. Am Heart J. 2008;156(2):e19. doi:10.1016/j.ahj.2008.04.031.

23. Gheorghiade M, Follath F, Ponikowski P, et al. Assessing and grading congestion in acute heart failure: a scientific statement from the acute heart failure committee of the heart failure association of the European Society of Cardiology and endorsed by the European Society of Intensive Care Medicine. Eur J Heart Fail. 2010;12(5):423-433. doi:10.1093/eurjhf/hfq045.

24. Núñez J, Llàcer P, García-Blas S, et al. CA125-Guided Diuretic Treatment Versus Usual Care in Patients With Acute Heart Failure and Renal Dysfunction. Am J Med. 2020;133(3):370-380.e4. doi:10.1016/j.amjmed.2019.07.041.

25. Felker GM, Anstrom KJ, Adams KF, et al. Effect of Natriuretic Peptide-Guided Therapy on Hospitalization or Cardiovascular Mortality in High-Risk Patients With Heart Failure and Reduced Ejection Fraction: A Randomized Clinical Trial. JAMA. 2017;318(8):713-720. doi:10.1001/jama.2017.10565.

26. Clerico A, Carlo Zucchelli G, Pilo A, Passino C, Emdin M. Clinical relevance of biological variation: the lesson of brain natriuretic peptide (BNP) and NT-proBNP assay. Clin Chem Lab Med. 


\section{Figures}

a

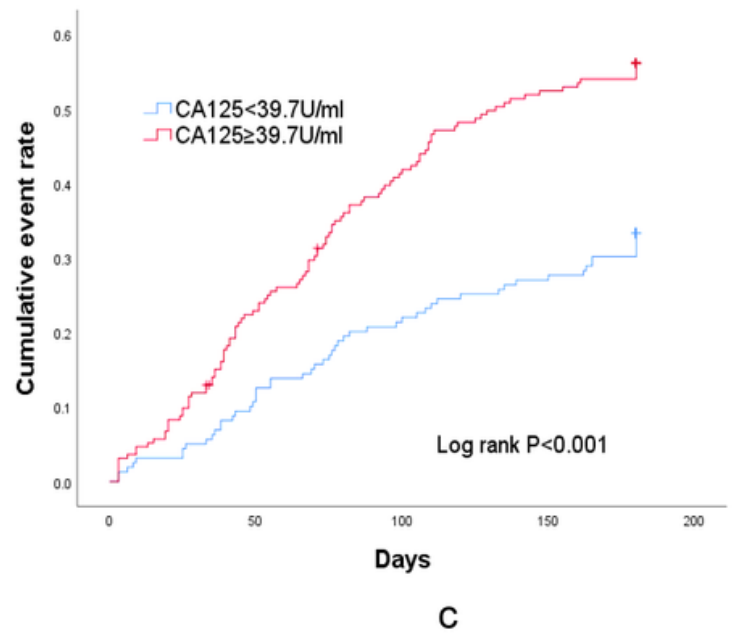

b

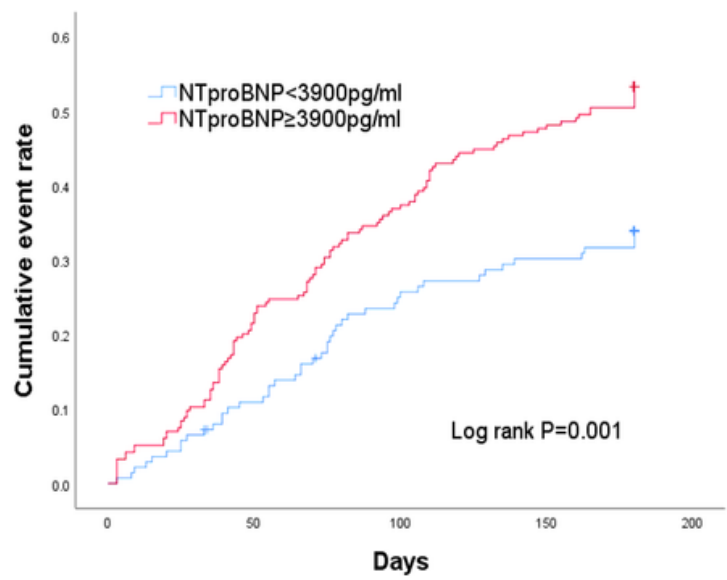

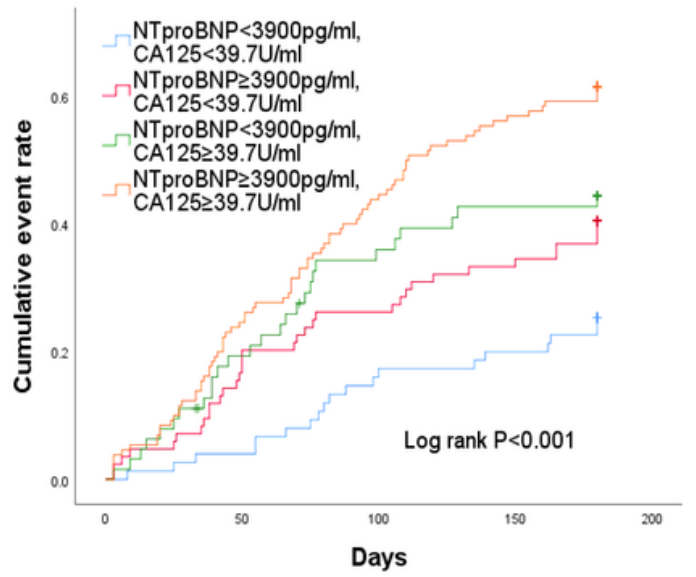

\section{Figure 1}

Kaplan-Meier estimates for 6-month combined endpoint of death/AHF rehospitalization stratified by CA125 (A), NTproBNP (B) and the combination of CA125 and NTproBNP (C). 